\title{
Zirconium Copper - a New Material for Use at Low Temperatures?
}

\author{
Adam L. Woodcraft \\ School of Physics and Astronomy, Cardiff University, Cardiff, 5 The Parade, Cardiff, CF24 3YB UK
}

\begin{abstract}
Cryogenic instruments usually require components with high thermal conductivity. Pure (non alloyed) copper offers very high conductivity, but is soft and not thus always suitable structurally. High conductivity beryllium copper $(\mathrm{BeCu})$ alloys are a common choice when copper is unsuitable since they offer a good compromise between hardness and thermal conductivity. These alloys contain a small $(<1 \%)$ amount of beryllium (along with a few percent of nickel or cobalt), with strength being obtained by precipitation hardening. An alternative precipitation hardened copper alloy is C15000 zirconium copper $(\mathrm{ZrCu})$, which contains $<0.2 \%$ zirconium with the remainder being copper. This material does not seem to be generally used in cryogenic systems, though hardness and strength are comparable to $\mathrm{BeCu}$. Moreover, the lower impurity content suggests that the thermal conductivity should be higher than for $\mathrm{BeCu}$. I present residual resistivity measurements suggesting that $\mathrm{ZrCu}$ does indeed possess considerably higher conductivity than $\mathrm{BeCu}\left(19 \mathrm{Wm}^{-1} \mathrm{~K}^{-1}\right.$ at $1 \mathrm{~K}$ compared to approximately $2 \mathrm{Wm}^{-1} \mathrm{~K}^{-1}$ ), and should therefore be a very useful material. A method is given for extrapolating the low temperature thermal conductivity values to temperatures up to $300 \mathrm{~K}$; good agreement is seen with room temperature data-sheet values. This extrapolation method is shown to also be applicable to $\mathrm{BeCu}$ and thus presumably other dilute copper alloys, and is used to give recommended values for coin silver and various BeCu alloy types, including C17510 and C17200.
\end{abstract}

To appear in the proceedings of the 24th International Conference on Low Temperature Physics, Orlando, Florida, USA, 10-17th August 2005. (American Institute of Physics, Suite 1N01, 2 Huntingdon Quadrangle, Melville, NY, USA

Keywords: thermal conductivity, cryogenic, zirconium copper, copper alloy, precipitation hardened, prediction, resistivity PACS: $72.15 . \mathrm{Eb}$

Cryogenic systems often require components with high thermal conductivity. These are usually constructed from pure copper, but alloys are used in some situations because copper is too soft. High conductivity beryllium copper $(\mathrm{BeCu})$ alloys are a common choice; these alloys contain a small $(<1 \%)$ amount of beryllium along with a few percent nickel or cobalt. Strength is obtained by precipitation hardening, and the low impurity levels provide a significantly higher conductivity than for most alloys of copper (or, indeed, any other material), offering a good compromise between strength and thermal conductivity.

Another precipitation hardened copper alloy which is commercially available is zirconium copper $(\mathrm{ZrCu})$, which contains $<0.2 \%$ zirconium. Hardness and strength are comparable to (though lower than) $\mathrm{BeCu}$, and the lower impurity content suggests that the cryogenic thermal conductivity should be higher than for $\mathrm{BeCu}$. This conclusion is supported by conductivity measurements at room temperature. The use of $\mathrm{ZrCu}$ also avoids the health hazards associated with $\mathrm{BeCu}$. However, there do not seem to be any reported measurements at cryogenic temperatures. Samples of $\mathrm{ZrCu}$ were therefore obtained for measurement (Outokumpu [1] material ZrK015, UNS code C15000; the composition is $0.12-0.20 \% \mathrm{Zr}$ by weight with the remainder being copper). The temper is unknown, but it is presumably in the precipitation hardened state. A test piece with a length of $140 \mathrm{~mm}$ and $32 \mathrm{~mm}^{2}$ cross-section was machined from the material. The electrical resistivity was measured at room temperature and $4.2 \mathrm{~K}$ (immersed in liquid helium) using a 4-wire DC method (AC measurements are subject to errors caused by wiring inductance).

The voltage was measured with a digital voltmeter. The current was provided by a magnet power supply ideal for such purposes because it is designed to output large, controlled currents through a low resistance using positive and negative polarity to eliminate the effect of thermal emfs. Ramping the current (under computer control) enabled any heating of the sample due to the measurement current to be observed; no such heating was seen. The measurement system has been checked by making measurements at low currents with a different current source and voltmeter; good agreement was seen.

The measured resistivity was $18.3 \mathrm{n} \Omega \mathrm{m}$ at $300 \mathrm{~K}$, in good agreement with the manufacturer's value of $18.7 \mathrm{n} \Omega \mathrm{m}$. The value at $4.2 \mathrm{~K}$ was $1.32 \mathrm{n} \Omega \mathrm{m}$, giving a residual resistivity ratio (RRR) value of 13.8 . The thermal conductivity can be calculated from the resistivity via the Wiedemann-Franz law. This is generally found to be accurate at liquid helium temperatures for pure copper, and also appears to be valid for $\mathrm{BeCu}$ [2]. We would thus expect it to be appropriate for $\mathrm{ZrCu}$. The resulting low temperature thermal conductivity is $19 \mathrm{Wm}^{-1} \mathrm{~K}^{-1}$ at a temperature of $1 \mathrm{~K}$, varying linearly with temperature.

The linear temperature dependence does not continue to room temperature. For pure copper, a set of equations 


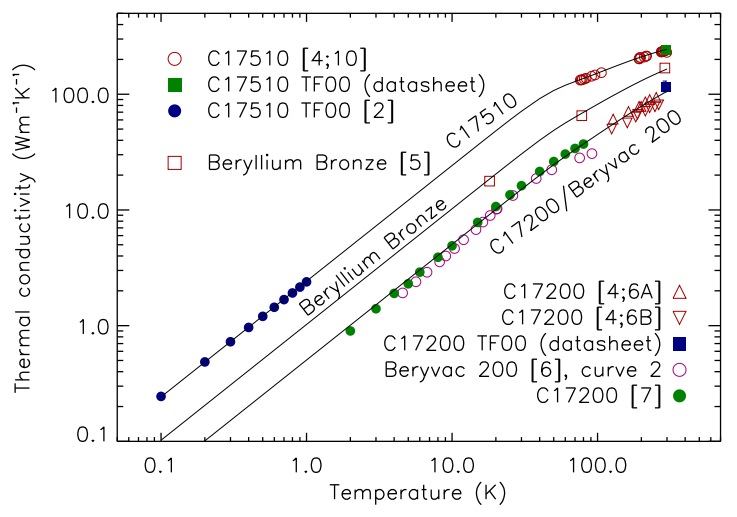

FIGURE 1. Measured thermal conductivity values for various dilute copper alloys (datapoints), along with fits using the equations from Ref. [3] (lines). References are given in square brackets; numbers following a semicolon identify different samples from a given reference.

have been derived that allow the thermal conductivity to be predicted between arbitrarily low temperatures and room temperature and above [3], based on the conductivity at a single temperature. These equations are accurate to approximately $10 \%$, and were derived for samples of approximately $\mathrm{RRR}=20$ and above.

However, they appear to be valid for $\mathrm{BeCu}$, with RRR values close to 1 . This is demonstrated in Fig. 1, where measured conductivity values [2,4-7] are compared to fits using the equations from Ref. [3]. It should be noted that in many cases the temper of the sample was not stated, and that different measurements on the same material should thus not necessarily agree perfectly. The excellent agreement for $\mathrm{C} 17510(0.2-0.6 \% \mathrm{Be}, 1.4-2.2 \% \mathrm{Ni})$ may thus be to some extent fortuitous. Results for 'high strength' BeCu alloys such as $\mathrm{C} 17200$ (1.8-2\% Be, Co + $\mathrm{Ni} \min 0.2 \%$, max $0.6 \%$ ) are shown; these have a higher beryllium content and are harder and stronger than the high conductivity alloys, but have poorer thermal and electrical conductivity. The measurements from Ref. [6] were made for a variety of heat treatments; the dataset corresponding best to the other measurements was chosen.

This good agreement suggests that the equations should also be valid for $\mathrm{ZrCu}$. It should be noted that using expressions for pure metals to fit the conductivity of alloys does not always work. For aluminium, the expressions for pure aluminium $(R R R>10)$ require modification for aluminium alloys [8].

Figure 2 shows the predicted conductivity values for $\mathrm{ZrCu}$. The room temperature value of $370 \mathrm{Wm}^{-1} \mathrm{~K}^{-1}$ is in excellent agreement with the data-sheet value of approximately $376 \mathrm{Wm}^{-1} \mathrm{~K}^{-1}$ (shown on the graph as a filled point). Values for other copper based materials

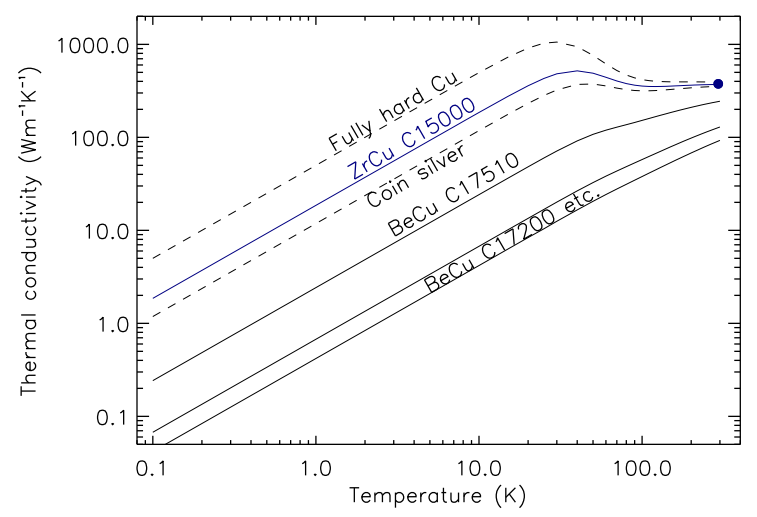

FIGURE 2. Recommended conductivity values for $\mathrm{ZrCu}$ and other coppers. Dashed lines are approximate.

with similar conductivity are also given in Fig. 2 . The curve labelled "C17200 etc." shows the likely range of values for high strength $\mathrm{BeCu}$ alloys [6]. Low temperature measurements for brass and bronze also fall into this range [2]. Values for annealed coin silver (90\% silver, $10 \%$ copper) [9] and fully hard (cold worked) pure copper [10] are also shown, based on low temperature measurements. These should be taken as an indication only since the conductivity can vary significantly depending on the exact treatment and the initial purity. While fully hard pure copper has a similar hardness to $\mathrm{ZrCu}$, and could be substituted in many cases, it is clear that $\mathrm{ZrCu}$ could be a very useful material for cryogenic use.

I would like to thank F. C. Gannaway for bringing this material to my attention, and Outokumpu for providing the samples.

\section{REFERENCES}

1. Outokumpu Sales Oy (UK), Regency Chambers, Regency Arcade, 154/156 Parade, Leamington Spa, UK.

2. A. Woodcraft, R. V. Sudiwala, and R. S. Bhatia, Cryogenics 41, 603-606 (2001).

3. J. G. Hust, and A. B. Lankford, National Bureau of Standards, Boulder, Colorado, 1984, NBSIR 84-3007.

4. N. J. Simon, E. S. Drexler, and R. P. Reed, NIST Monograph 177, 1992.

5. Y. S. Touloukian, P. E. Liley, and S. C. Saxena, Thermophysical properties of matter. Vol. 1. Thermal conductivity: metallic elements and alloys, Plenum, 1970.

6. V. Gröger, and F. Stangler, Zeitschrift für Metallkunde $\mathbf{7 2}$, 487-9 (1981).

7. R. Berman, E. L. Foster, and H. M. Rosenberg, Brit. J. App. Phys. 6, 181-182 (1955).

8. A. L. Woodcraft, Cryogenics 45, 421-431 (2005).

9. C. T. V. Degrift, Physica $B \& C$ 107, 605-6 (1981).

10. K. T. Hartwig, and P. G. Appius, Proceedings of the 8 th international cryogenic conference, 1980, pp. 720-725. 